\title{
AlfaBeta: Um jogo com realidade virtual para auxiliar a alfabetização e o aprendizado da grafia correta de palavras
}

\author{
Adilmar C. Dantas ${ }^{1}$, Sara L. de Melo ${ }^{2}$, Michel S. Xavier ${ }^{1}$, Guilherme B. dos Santos ${ }^{1}$ \\ Ananda R. dos Santos ${ }^{1}$, Heidie S. Torres ${ }^{1}$, Celso A. de Souza Barros Gonçalves ${ }^{1}$ \\ Marcelo Z. do Nascimento ${ }^{1}$ \\ ${ }^{1}$ FACOM - Faculdade de Ciência da Computação \\ Universidade Federal de Uberlândia \\ Av. João Naves de Ávila, 2121-38408-100 Uberlândia, MG, Brasil \\ ${ }^{2}$ FEELT - Faculdade de Engenharia Elétrica \\ Universidade Federal de Uberlândia \\ Av. João Naves de Ávila, 2121-38408-100 Uberlândia, MG, Brasil \\ email\{akanehar,saraluziamelo,miichelsx, brilhquo, anandaroberta25, heidietorres \\ celsoandre2002, marcelo.zanchetta\}@gmail.com
}

\begin{abstract}
Resumo. Esse trabalho apresenta um jogo com realidade virtual para auxiliar professores elou tutores durante o processo de alfabetização. No jogo, o estudante é inserido em um ambiente imersivo com objetos modelados tridimensionalmente e existentes no mundo real juntamente com as letras do alfabeto, para que ele forme corretamente o nome do objeto solicitado. Além disso, o jogo possui uma seção para o treino da grafia correta das palavras de maneira divertida e descontraída proporcionada pelo uso da realidade virtual. A ferramenta foi disponibilizada gratuitamente para coleta de dados e feedback dos usuários.
\end{abstract}

\section{Cenário de Uso}

Segundo pesquisa realizada no trabalho de [MORTATTI 2006], muitas escolas públicas do estado e dos municípios apresentam limitações em relação ao processo de alfabetização. Nesse mesmo caminho, existe um elevado número de alunos não alfabetizados em cada uma das séries do ensino fundamental. Em muitos casos, parte desses estudantes apenas são alfabetizados ao longo da vida escolar.

O uso de tecnologias, mas especificamente, a Realidade Virtual (RV), vêm sendo empregadas em diversas áreas do conhecimento. De maneira geral, a RV pode ser considerada a junção de três conceitos: imersão, interação e envolvimento [Morie 1994]. Isso tem permitido pesquisas interdisciplinares com uso de sistemas computacionais e teleoperação [Brandão et al. 1998].

A ideia de imersão empregada RV permite explorar a sensação do usuário estar presente dentro do próprio ambiente. Isso tem possibilitando ao usuário fazer parte desse ambiente. Essa característica, normalmente, é alcançada com o uso de dispositivos, como salas de projeção e capacetes ou óculos de RV. Esses dispositivos são interconectados a outros dispositivos, como som, localização, movimentação do usuário no espaço e dispositivos reativos, para incrementar a sensação de imersão [Begault and Trejo 2000]. Já a interação esta relacionada a capacidade do sistema computacional, seja um computador ou smartphone, de detectar as entradas do usuário e emitir respostas dentro do ambiente 
VII Congresso Brasileiro de Informática na Educação (CBIE 2018)

Anais dos Workshops do VII Congresso Brasileiro de Informática na Educação (WCBIE 2018)

em tempo real. Em geral, esse é um dos aspectos que mais atraem jogadores, como por exemplo, a mudança de cenários e ações realizadas por meio de comandos e interações. $\mathrm{O}$ último conceito é o envolvimento, o qual trata a capacidade de envolvimento do usuário para realizar uma tarefa. Esse envolvimento pode ser passivo, como ler um livro, ou ativo, como executar atividades em um jogo com outro usuário. A RV pode ser empregada nos dois casos, pois permitir ao usuário explorar esses ambientes por meio das interações.

Atualmente, em nosso cotidiano, tem-se empregado diversas ferramentas computacionais com objetivo de explorar conhecimentos. Exemplos de aplicações são os jogos corporais e jogos de cores, os quais apresentam uma combinação de sons o qual permite ao usuário despertam a curiosidade. Isso contribui na aquisição de novos conhecimentos. De acordo com [CLARK 1974], o uso de jogos empregados em educação é bem reconhecida e perceptível. No trabalho [Júnior et al. 2017], os autores apresentaram um exemplo da utilização de RV para o ensino de conceitos programação, dando destaque ao potencial que as tecnologias tem proporcionado quando bem elaboradas e aplicadas no contexto educacional. A utilização de jogos na educação pode trazer uma série de benefícios, principalmente, conceitos relacionados a intuição, a solução de problemas e comportamento intersocial. A utilização de jogos tem contribuído no contexto educacional, mas as dificuldades enfrentadas pelos educadores no momento da alfabetização das crianças tem-se apresentando com um grande desafio para área.

Para tanto, esse trabalho apresenta um jogo denominado Alfabeta para ser empregado no processo de alfabetização, proporcionando aos estudante, um momento descontraído e divertido na aprendizagem. Essa ferramenta permite auxiliar professores e pode ser utilizado tanto em sala de aula quanto em casa, com os pais ou responsáveis pelo estudante.

\section{Desenvolvimento}

Para o desenvolvimento do jogo Alfabeta foi realizado uma avaliação de tecnologias empregadas em RA. Os recursos e tecnologias web apresentam vantagens que estão relacionados ao fato de permitirem a utilização em dispositivos móveis, pois possuem recursos com bom desempenho e não possuem custo elevado. Nesse aplicativo foi utilizado o Jquery Mobile, que consiste em ferramenta de interface baseado no HTML5, versão 5.2, o qual possibilita a criação de aplicações acessíveis [jQuery 2017] para qualquer dispositivo que possua um navegador web. Essas tecnologias permitem o uso de novos atributos, comportamentos e web sites dinâmicos [Mozilla 2018]. Essas características contribuem no desenvolvimento de interface do jogo responsável pela apresentação visual ao usuário.

A modelagem dos objetos 3D foi realizada com o software livre de código aberto Blender. O Blander é uma plataforma de criação que da suporte a diversas extensões, manipulação, animação, simulação, renderização, composição e rastreamento de movimento, edição de vídeo e criação de jogos [Blender 2018]. Na Figura 1 é apresentado um modelo desenvolvido para o jogo.

Neste trabalho foi empregado o framework, de código aberto, A-FRAME para o desenvolvimento dos ambientes do jogo. O A-FRAME é uma estrutura de sistema de componentes empregadas para criar cenas 3D e WebVR usando HTML, o qual é mantido pela Mozilla e pela comunidade WebVR [A-FRAME 2018]. Essa ferramenta é muito empregada na construção de experiências de RV. 
VII Congresso Brasileiro de Informática na Educação (CBIE 2018)

Anais dos Workshops do VII Congresso Brasileiro de Informática na Educação (WCBIE 2018)
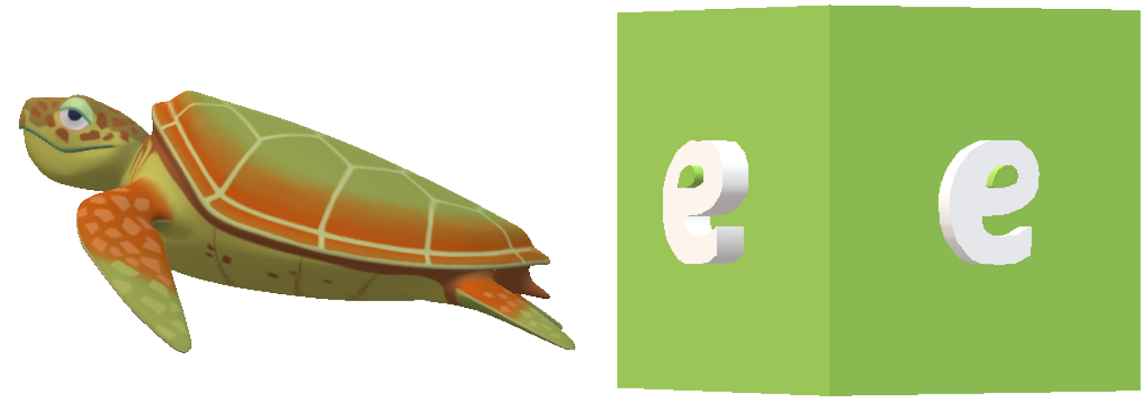

Figura 1. Exemplo de objetos construídos em 3D para utilização no jogo.

Para avaliar o funcionamento e desempenho do framework em dispositivos móveis foram selecionados seis dispositivos móveis: dois aparelhos de alto custo, dois de custo médio e dois de baixo custo de mercado. Foi avaliado o desempenho na reprodução do jogo e limitações para executar o jogo. A Tabela 1 traz as informações detalhadas em relação a avaliação dos aparelhos empregados.

Tabela 1. Análise do jogo em dispositivos móveis com diferentes recursos computaconais.

\begin{tabular}{lll}
\hline Aparelho & Sistema Operacional & Resultado \\
\hline Motorola Z2 Play & Android 7.1 ou superior & Funcional sem limitações. \\
Iphone 6s & iOS 10.1 ou superior & Funcional sem limitações. \\
Motorola G5 Plus & Android 7.1 ou superior & Funcional sem limitações. \\
LG K10 & Android 6.0 ou superior & Funcional sem limitações. \\
Samsung Galaxy J7 & Android 7.1 ou superior & $\begin{array}{l}\text { Não funcional com limitações, devido a } \\
\text { ausência de sensores como o giroscópico. }\end{array}$ \\
Asus ZenFone 2 & Android 6.0 ou superior & Funcional sem limitações.
\end{tabular}

O experimento sobre esses aparelhos permitiu classificá-los em relação a funcionalidade para jogos com uso do framework. Essa investigação mostrou em aparelhos de hardware moderado a execução foi bem-sucedida para a criação da experiência virtual necessária em jogos.

Para garantir sempre o bom funcionamento do jogos no dispositivos móveis com objetivo ter desempenho adequado, os objetos 3D foram armazenados em um servidor web juntamente como os recursos gráficos, como por exemplo, imagens, áudio e conjunto de scripts. Na Figura 2 o procedimento de usuário e servidor é exemplificado. Essa estratégia exige na primeira vez uma boa conexão para que os objetos possam ser carregados ou, em conexões baixas, poderá demorar um tempo para carrega-los. Em conexões futuras, isso não irá acontecer devido ao fato que os objetos ficam alocados no cache.

Essa abordagem permite que as ações relacionadas a reprodução do cenário, áudio, controles e os elementos de RV sejam executados pelo navegador do usuário. Esse aplicativo faz o gerenciamento de memória e recursos de forma a permitir o adequado funci- 


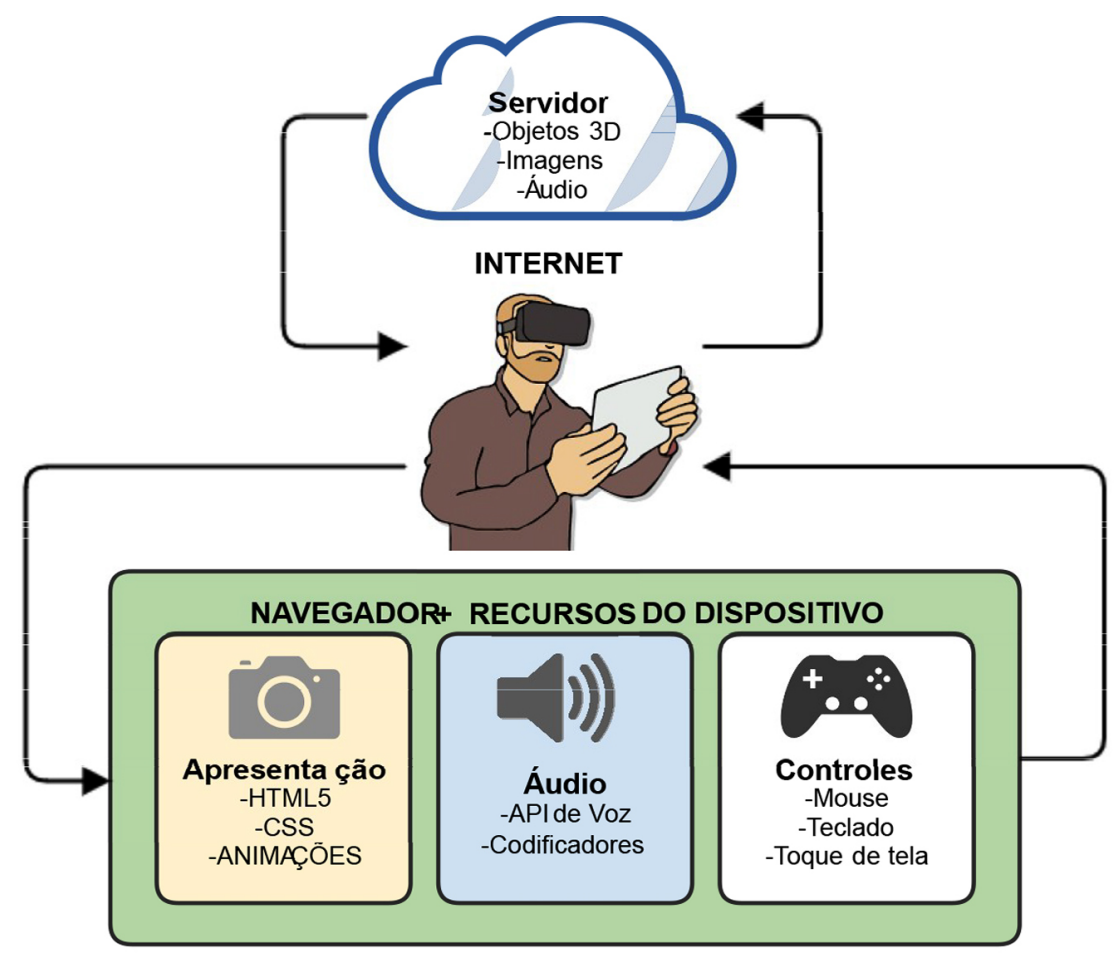

Figura 2. Diagrama da estratégia aplicada no aperfeiçoamento do desempenho do jogo em dispositivos móveis.

onamento do jogo.

\section{Apresentação do software}

O jogo desenvolvido pode ser facilmente acessado através de um endereço web ou por meio do aplicativo. A execução pode ocorrer em computadores com uso de teclado e o mouse, para realizar as operações e comando no jogo, ou pela virtualização, em aparelhos móveis, com o auxílio de óculo de RV.

O jogo proposto não é limitado apenas a quem possui um óculos de RV. Para permitir a imersão ao jogo, o aplicativo proposto possui uma opção de gerar o molde do Google Cardboard, um óculos a ser construído em papelão para experiências em RV [Google 2018]. Na Figura 3 é apresentado o óculos empregado neste estudo. A utilização desse óculos além de contribuir para a redução dos custos para emprego em escola público pode contribuir com o meio ambiente reutilizando materiais que seriam descartados. Se o usuário optar por não montar o óculos de papelão. A empresa Google oferece a preços acessíveis para projetos relacionados a RV.

Na Figura 4 é apresentado a primeira interface responsável pela apresentação de RV para o aluno. Na primeira interface do jogo é apresentado as instruções para o aluno. As informações e interações do jogo ocorrem por meio de áudio. Os áudios são falas que foram processadas pelo serviço de síntese fornecido pelo Google. Nessa interface, o estudante tem o primeiro contato com a RV e pode treinar os movimentos da cabeça com uso do óculos de RV junto com o dispositivo móvel.

A segunda interface do jogo, o usuário deve ouvir uma palavra produzida pelo sintetizador de voz. Essa palavra pode ser um nome de um animal ou de um objeto e 
VII Congresso Brasileiro de Informática na Educação (CBIE 2018)

Anais dos Workshops do VII Congresso Brasileiro de Informática na Educação (WCBIE 2018)

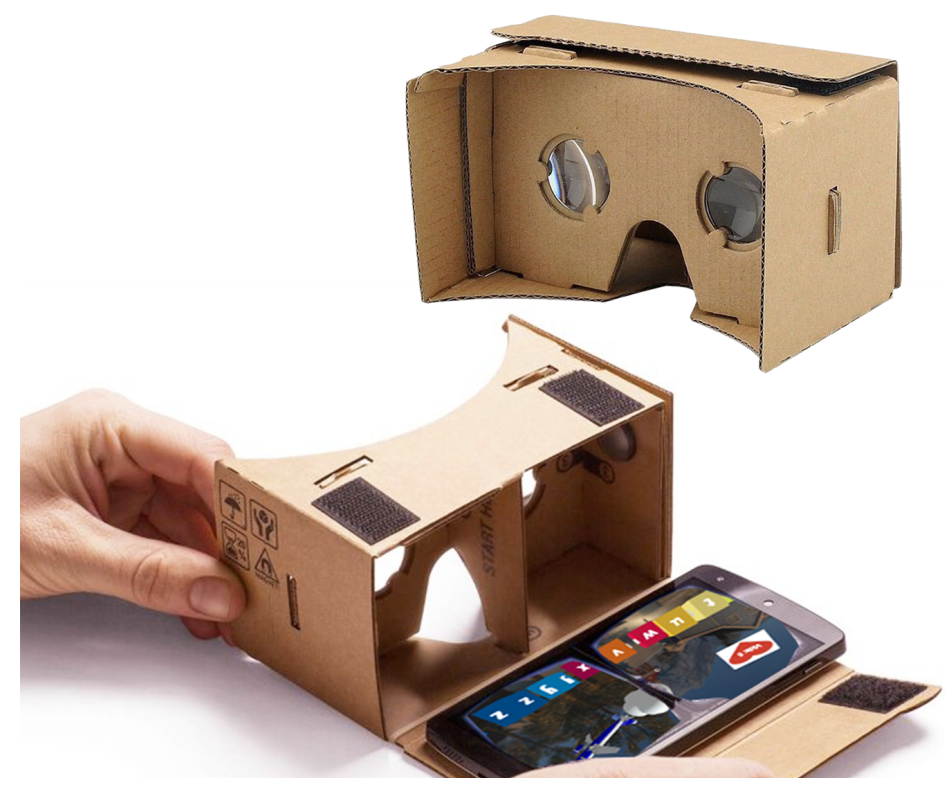

Figura 3. Óculos do Google Cardboard utilizado para executar o jogo em RV.

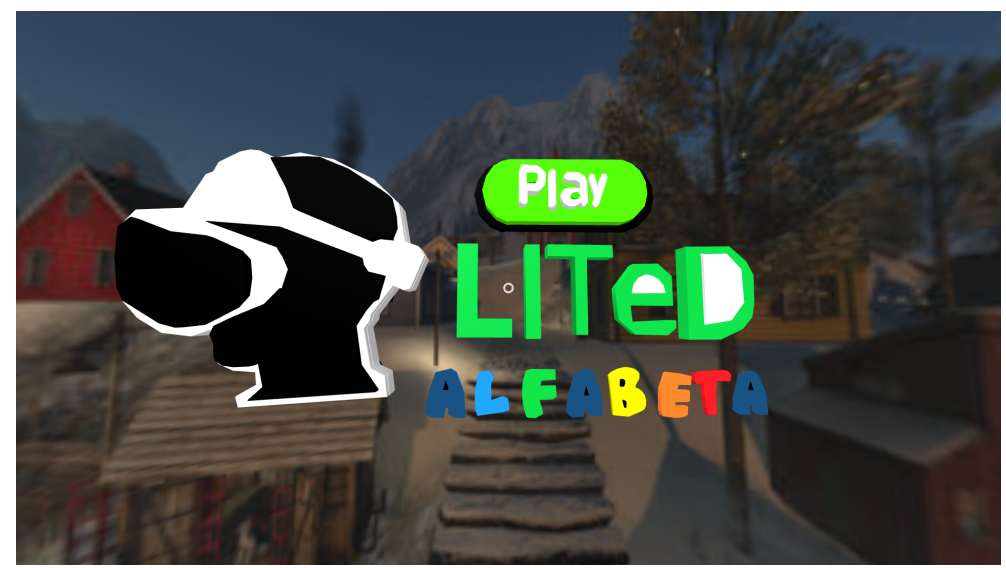

Figura 4. Interface do jogo responsável pela iniciação das instruções permitindo a imersão do aluno em ambiente de RV.

o aluno deve encontrar as letras que formam aquela palavra. As letras estão dispostas em torno de todo ambiente virtual, conforme mostrado na Figura 5 a palavra "avião". Nessa interface também é apresentado o objeto 3D e o usuário pode selecionar para ouvir novamente a palavra para que posso procurar as letras.

Há um painel informativo com o número de tentativas na interface. O jogo permite até cinco tentativas em que a cada letra marcada que não faz parte da palavra e decrementado uma tentativa do usuário. O número de tentativas é reiniciado a cada nova palavra. A cada nova palavra permite explorar as letras do alfabeto reforçando o aprendizado do aluno. Com o objetivo de fornecer um feedback para o educador, cada palavra que apresenta dificuldade para o aluno são registradas em um banco de dados. $\mathrm{O}$ banco de dados foi projetado usando a linguagem SQL, um tipo relacional bastante popular para sistemas online [Delisle 2006]. Após uso do jogo um relatório é apresentado com as informações sobre o desempenho do estudante para o educador. 


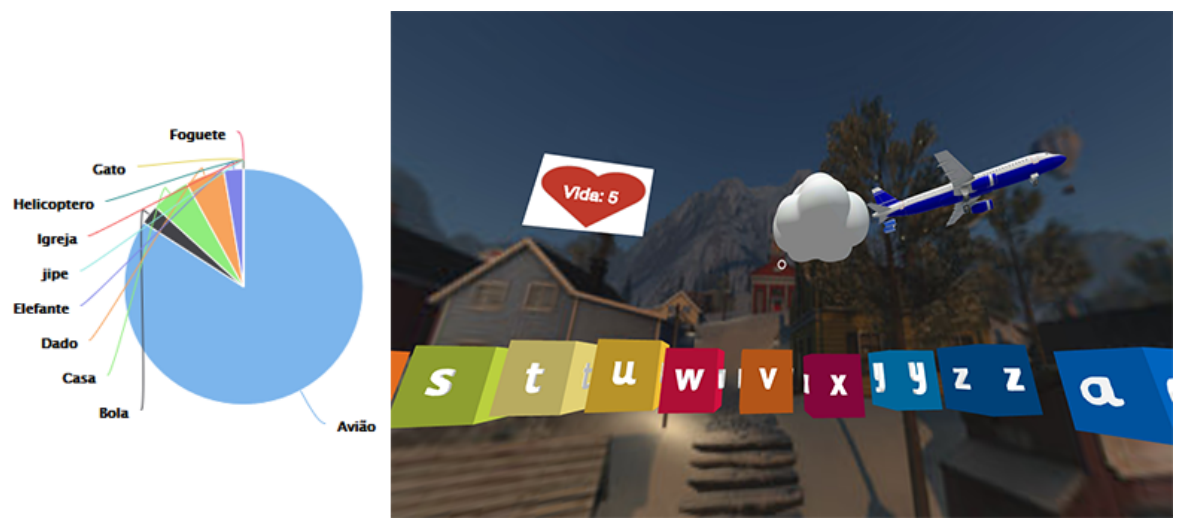

Figura 5. Gráfico de desempenho dos usuários e ambiente virtual do jogo: nesse cenário o objetivo é encontrar as letras da palavra avião.

\section{Considerações finais}

Para avaliar a usabilidade e a aceitação do uso de jogos, que utilizam RV, no aprendizado pretende-se aplicar o jogo aos estudantes de instituição de ensino público. A sala selecionada na instituição é composta por 23 alunos do $2^{\circ}$ ano do ensino fundamental. O método de avaliação sera composto por duas etapas. Na primeira os alunos deverão resolver uma atividade elaborada juntamente com o docente responsável. Essa primeira atividade tem como objetivo avaliar o conhecimento inicial dos alunos referente a grafia correta das palavras.

Após a avaliação, os alunos serão submetidos a praticarem as mesmas palavras por meio do jogo com a intenção de colaborar no processo de aprendizagem das palavras. No final dessa etapa ocorrera uma análise dos conceitos adquiridos durante a aplicação no ambiente escolar. Os dados de desempenho dos alunos serão comparados entre os resultados iniciais e após uso dessa ferramento com objetivo de investigar as contribuições e limitações no contexto educacional.

\section{Referências}

A-FRAME (2018). Game development@ONLINE.

Begault, D. R. and Trejo, L. J. (2000). 3-d sound for virtual reality and multimedia.

Blender (2018). The software@ONLINE.

Brandão, E. J. R., Trentin, M. A., Lebedeff, T. B., Mortari, M. I., Oro, N. T., and Pasqualotti, A. (1998). A realidade virtual como proposta de ensinoaprendizagem de matemática para deficientes auditivos-rv_peamda. In anais do IV Congresso Ibero Americano de Informática Educativa. URL: lsm. dei. uc. pt/ribie/docfiles/txt200342319516197. PDF, descarregado em, volume 12, page 2003.

CLARK, C. (1974). Jogos simulados-estratégia e tomada de decisão. Jogos Simulados: Estratégia e Tomada de Decisão.

Delisle, M. (2006). Creating your MySQL Database: Practical Design Tips and Techniques. Packt Publishing Ltd.

Google, C. (2018). Experience virtual reality in a simple, fun, and affordable way@ONLINE. 
VII Congresso Brasileiro de Informática na Educação (CBIE 2018)

Anais dos Workshops do VII Congresso Brasileiro de Informática na Educação (WCBIE 2018)

jQuery (2017). Jquery mobile about@ONLINE.

Júnior, G. S., Ramos, T., Fontes, L., Dorea, F., Goulart, F., Júnior, G., and Menezes, J. (2017). Escapevr: Labirinto em realidade virtual para auxiliar na aprendizagem de algoritmos de busca. In Anais dos Workshops do Congresso Brasileiro de Informática na Educação, volume 6, page 363.

Morie, J. F. (1994). Inspiring the future: merging mass communication, art, entertainment and virtual environments. ACM SIGGRAPH Computer Graphics, 28(2):135-138.

MORTATTI, M. d. R. L. (2006). História dos métodos de alfabetização no brasil. Seminário Alfabetização e Letramento em Debate. Brasília.

Mozilla (2018).Html5@ONLINE. 